\title{
Sons al balcó: Soundscape Map of the Confinement in Catalonia ${ }^{\dagger}$
}

\author{
Rosa Ma Alsina-Pagès $\left.{ }^{1, *} \mathbb{(}\right)$, Ferran Orga ${ }^{1}\left(\mathbb{D}\right.$, Roger Mallol $^{1}\left(\mathbb{D}\right.$, Marc Freixes $^{1}\left(\mathbb{D}\right.$, , Xavier Baño $^{2}$ \\ and Maria Foraster ${ }^{3}$ (D)
}

1 Grup de Recerca en Tecnologies Mèdia (GTM), La Salle-URL, C/Quatre Camins, 30, 08022 Barcelona, Spain 2 Research and Transference Office, La Salle-URL, C/Sant Joan Baptista de La Salle, 42, 08022 Barcelona, Spain 3 ISGlobal, Parc de Recerca Biomèdica de Barcelona (PRBB), Doctor Aiguader, 88, 08003 Barcelona, Spain

* Correspondence: rosamaria.alsina@salle.url.edu; Tel.: +34-93-2902425

+ Presented at the 7th International Electronic Conference on Sensors and Applications, 15-30 November 2020; Available online: https:/ / ecsa-7.sciforum.net/.

Published: 15 November 2020

check for updates

\begin{abstract}
In this project, we aim to study the effect that the lockdown due to the COVID-19 pandemic has caused on the perception of noise in Catalonia. In Sons al Balcó, the research activities cohabit with the dynamic collaboration with citizens and other stakeholders to create social and environmental impact, to widen awareness and design tools to improve citizenship development and empowerment. The initial scientific hypothesis is that the annoyance coming from outdoor noise, minimized by the lockdown effect, could be associated with better perception of the soundscape by people. Sons al Balcó allows to validate this hypothesis in two different ways. On the one hand, by means of subjective questionnaires conducted to people living in pre-defined diverse acoustic areas (urban, suburban and rural environments), and on the other hand, by the use of objective measurements of the noise levels, and the study of the soundscape in these areas, using short pieces of video collected by citizens. For this purpose, we have designed an on line test to be conducted by any citizen aiming to contribute to this wide study for all the territory of Catalonia, both from rural areas and from cities. A communication campaign was conducted to reach a significant participation. During the lockdown, more than 350 questionnaires and videos were collected, and a first map of the soundscape of the confinement in Catalonia was depicted.
\end{abstract}

Keywords: soundscape; lockdown; urban; suburban; perception

\section{Introduction}

Every year, environmental noise causes 48,000 new cases of ischeamic heart disease and more than 12,000 deaths in Europe [1]. Even more, it generates chronic high annoyance to more than 22 million people, and chronic high sleep disturbance to more than 6.5 million [2]. These health estimates are based on the last WHO report [1], which presents recommendations regarding the equivalent levels of noise that should not be exceeded to protect health.

During the March-April-May 2020 lockdown, the soundscape of our homes changed drastically [3,4]. All that sound [5] associated with daily activity became almost non-existent, especially during the strictest closing weeks. Noise from road traffic [6], railroad noise, airport noise, as well as all that noise related to leisure, were substantially reduced in most of the analyzed cities $[7,8]$. On the contrary, the sound related to in-house activities and neighbours was increased. Also, the sound related to animals was more relevant; in some parts of big cities, birdsong was heard for the first time in years.

In this study, we aim to study the effect that the lockdown due to the COVID-19 pandemic has caused on the perception of noise in Catalonia by means of Citizen Science. Other similar projects 
have been conducted in the United Kingdom [9], Italy [10-12] in New York City [13] even worldwide with the goal of registering the exceptional soundscape conditions in all the cities [14]. The hypothesis is that the annoyance coming from outdoor noise, minimized by the lockdown, could be associated with greater well-being of people. This hypothesis will be validated on the one hand, by means of subjective questionnaires conducted to people living in pre-defined diverse acoustic areas (urban, suburban, rural environments), and on the other hand, by the use of objective measurements of levels of noise, and the study of the soundscape in that areas.

Our proposal is based on assessments of how citizens have experienced the changes in their acoustic environment -both the pleasant ones but also especially the annoyance factors-, as exceptionally they have spent much more time at home. We evaluate the citizens' perceptions together with the objective measurements, which should lead to the conclusions about the type of noise to avoid and the definition of several indicators as guidance to improve the well-being and quality of life in different geographical areas. The comparison between the samples collected during lockdown, and the possibility of carrying out more exhaustive collection of data in the post-lockdown, gives the opportunity to the work to compare several aspects never previously analysed or studied. We have experienced the longer ever period of cessation of industrial, commercial and educational activities in our environment, and the impact of this fact on people has never been modelled.

\section{Sons al Balcó and Social Impact}

Our proposal does not only seek scientific results, but also to evidence the essential role of research by means of some of its key aspects. From the basis of providing knowledge and novel technologies to solve challenges defined by humans, this project traces a path to expand the scientific results from the research activities in order to promote social development. With the main focus on the core research activities, we envision to make the project available to create a clear social and environmental impact in the places under study. Collaboration is a key aspect of research, and in this sense, we promote dynamic interactions with innovators, entrepreneurs and citizens in order to widen awareness, provide knowledge, create technologies and tools to improve the social development.

In order to extent our purpose, we face the challenge of focusing on cross-boundaries and putting our research results available to work with other interest groups from other disciplines like art, education, environment, health, citizenship and territory. We can provide outputs that can support the policies design, propose solutions and take actions in ecology and sustainability, urban planning and policies, social and health interventions, educational projects and scientific dissemination, even through artistic proposals to widen the scope of the public.

As a result of this approach implementation, we impulse the creation of a social and environmental impact directly related to several of the Sustainable Development Goals described by the United Nations [15] (UN) to promote prosperity while protecting the planet. Sons al Balcó contributes responding to seven of the seventeen goals defined: Life on Land, Climate Action, Sustainable Cities, Quality Education and Good Health and Well-being. Indirectly, due to the holistic vision of the work, we also support reaching the goals of Partnership and Reduced Inequalities. The aim of this project is that our research activity combines the deep scientific work that pursue build new knowledge, together with a open and interdisciplinary collaboration with key actors that facilitate our contribution to a social and environmental development.

\section{Data-Collection Campaign}

\subsection{Design of the First Recording Campaign}

During the COVID-19 lockdown, we performed a socio-acoustic digital participatory survey implemented in LimeSurvey [16] and distributed through the social media and press to all citizens of Catalonia. The used social media was twitter, where messages and continuous information was uploaded via our institutional accounts (@laSalleBCN and @ISGlobalorg) and the Sons al balcó project 
(@sonsalbalco) account. We had an outreach of 15,000 impressions and 400 engagements in the first tweet of the campaign; nevertheless, individual interaction of each engaged citizen was required to complete information on all areas of the map.

The survey included questions about socio-demographics, the quality of the residential soundscape and the individual positive and negative perception before and during the lockdown. Furthermore, the survey allowed participants to upload videos of their residential soundscapes and to characterize several types of sounds sources appearing in the video. The sound source categories that can be marked by the user include: car traffic, trains, airplanes, industry, construction, commercial activities, leisure, neighbours, pets, birds, water-related sounds and vegetation.

Thus, we envisage the use of the following quantitative data:

- Surveys to citizens about their perception of the soundscape before, during and after confinement (yet to be conducted) throughout Catalonia, with details of which are the most annoying sounds and quantifying this annoyance.

- Recordings collected thanks to the citizen science web platform during the lockdown and also afterwards (het to be conducted), in the so-called New Normality.

- Measurements of equivalent acoustic level $\left(L_{A e q}\right)$ carried out by sensors calibrated in the cities where they are available and operative, before, during and after the lockdown.

\subsection{Framework of the Data Collection}

Sons al Balcó required an application to launch an online survey including diverse response types, even video uploading (see Figure 1). This last option, though, is not included in the applications available on the web. The majority of online survey applications offer their services on their own servers, which limits the customisation options. Furthermore, these web services are not designed to video uploading requirements neither from a network bandwidth or storage point of view. Taking into account these requirements, LimeSurvey [16] was the solution adopted for the survey deployment. This platform does not only allow for the creation of online question-and-answer surveys with a wide variety of response formats, but their source code is also available so to be deployed in your own server. Specifically, we contracted an Amazon EC2 cloud computing instance running a Bitnami Stack for Limesurvey 4.2.3-0 on Ubuntu 16.04.6 LTS.

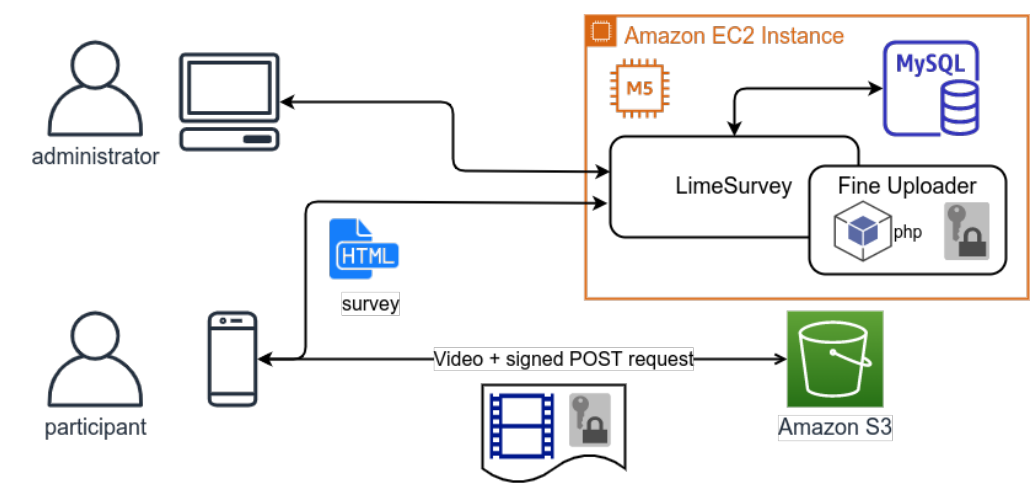

Figure 1. Online survey framework based on LimeSurvey [16], deployed on an Amazon EC2 instance with video uploading to an Amazon S3 bucket using Fine Uploader [17].

LimeSurvey offers a web platform to create and edit surveys. Once the survey is published, it is delivered to the participant in html format compatible with most of smartphone web browsers. Video can be recorded in web browser using the RecordRTC JavaScript library [18] based on WebRTC [19]. However, this option was discarded because the web browser asks permission to access the device camera, which can be perceived by participants as intrusive. Instead, an input $\mathrm{html}$ tag with the attribute accept="video/*" was included in the survey. This tag is interpreted by the smartphone, which launches the camera application. The downside of this approach is that 
videos are recorded in high definition, thus producing very large files. The option of uploading a prerecorded video was also included by adding a second input tag without the accept attribute. In this case, the smartphone launches a file browser.

In order to reduce data traffic to the EC2 instance, it was decided to directly upload the videos from the device web browser to an Amazon S3 bucket. To this end, Fine Uploader library was used [20]. A php service runing on the EC2 instance manages and signs the requests that allow the access to the S3 bucket [17]. JavaScript code to manage video uploading was embedded in the survey. This code uses Fine Uploader functions to ask for a signed POST request and send it to the S3 bucket together with the video. Besides the aforementioned characteristics, Fine Uploader also allows for file partitioning and auto-resume of interrupted uploads.

\section{Drawing the Map of the Confinement in Catalonia}

The questionnaire was open from 20 May 2020, until 12 June 2020. In total, 366 volunteers completed the questionnaire and uploaded their video. We analyzed the collected data, and decided to discard one entry because having an undetermined name ("La Ametlla"). Also, there was an entry from "Gavà Mar", which was labelled as "Gavà". Figure 2 shows a visual representation of the geographical distribution of the participating citizens and the distribution in the Catalan territory.

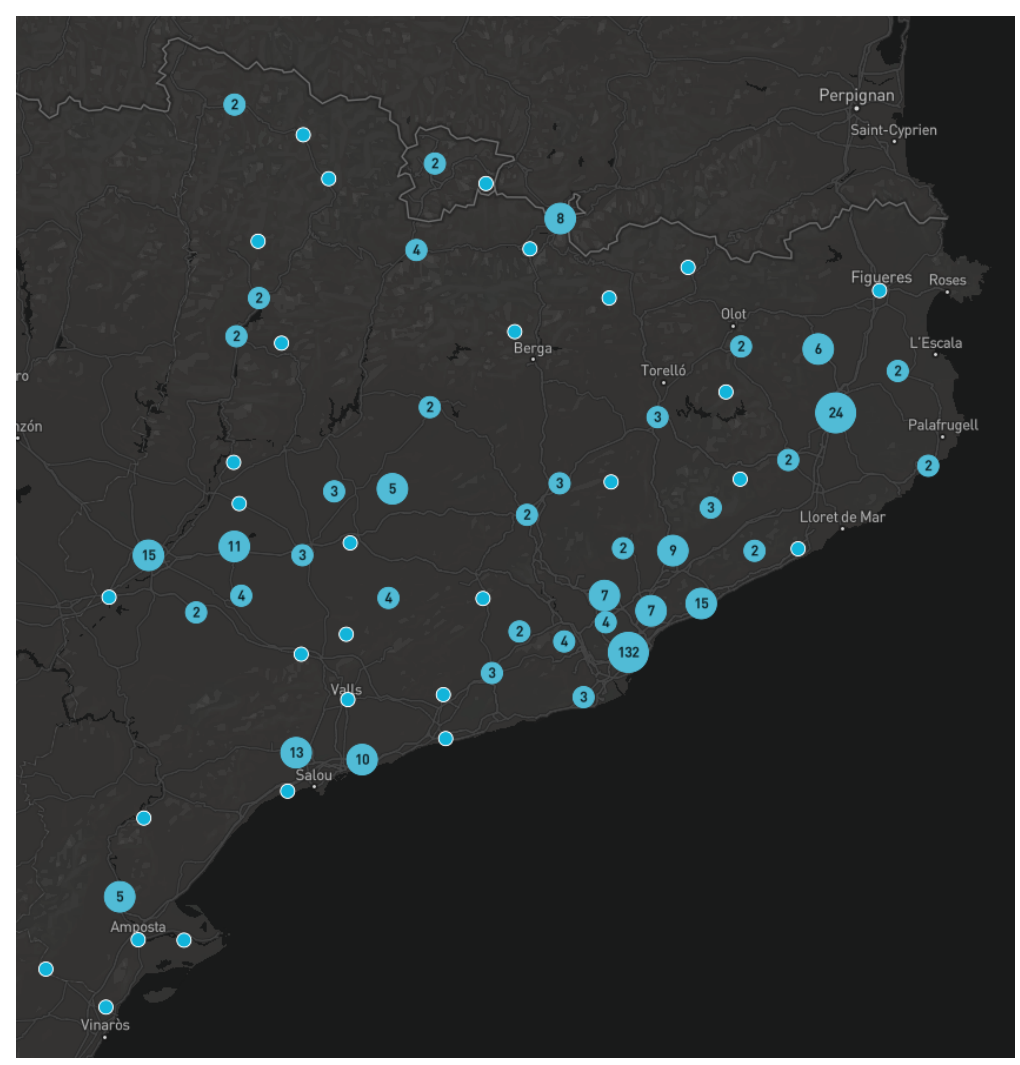

Figure 2. Schematic of the geographical distribution of the participating cities.

The following table shows the distribution of the participating locations and recorded videos based on the number of inhabitants per location. To mention, the number of inhabitants per city is based on official data from 2019 for the majority of the cities (Institutd'EstadísticadeCatalunya,https: //www.idescat.cat/pub/?id=aec\&n=1, accessed 15 October 2020 ), with some exceptions where data from prior years had to be used because of the lack of updated data. 
Table 1. Number of participating locations and recorded videos across different ranges of inhabitants per city.

\begin{tabular}{lccccccc}
\hline & $<\mathbf{5 0 0}$ & $\mathbf{5 0 1 - 2 0 0 0}$ & $\mathbf{2 0 0 1 - 5 0 0 0}$ & $\mathbf{5 0 0 1 - 1 0 , 0 0 0}$ & $\mathbf{1 0 , 0 0 1 - 5 0 , 0 0 0}$ & $\mathbf{> 5 0 , 0 0 0}$ & Total \\
\hline Number of locations & 17 & 21 & 18 & 24 & 34 & 18 & 132 \\
Number of recorded videos & 20 & 26 & 26 & 34 & 61 & 198 & 365 \\
\hline
\end{tabular}

\section{Discussion}

We propose to analyse the collected data considering several approaches: (i) geographical descriptions, (ii) innovative acoustic event detection algorithms as well as computer vision algorithms to the video contents to characterize the soundscapes, (iii) statistical analyses of the differences in annoyance before, during and after the lockdown, with a special focus in the sounds highlighted by citizens. Also, we will consider confusion variables that can play their role when evaluating annoyance. For this reason, we propose a comparison between different acoustic environments in the same place, and also consider the pleasantness of several usual types of sound in the questionnaire. The results of these analyses will lead the team to describe a set of indicators to compare the well-being of citizens in terms of acoustic comfort for the three different moments of the analysis.

In this sense, we aim to provide a healthscape; beyond the concept of soundscape, combining the data collected by citizens and -if possible - acoustic measurements obtained by sensors, by means of its combination with the annoyance or pleasantness experience described by citizens. The soundscape can be described even in various places—urban, suburban, and rural-but the incorporation of perceptions of annoyance by people, for which we kindly thank volunteers for their collaboration, gives the possibility of mapping a more exhaustive model of the different geographies under study in terms of measured values and annoyance perception.

Our project is innovative in two areas; firstly, because it responds to the exceptionality of so many weeks of confinement at home. The circumstance of the widespread shutdown of non-essential services and industry has positioned the acoustic environment at a noise level values that had never been recorded before, especially in certain urban areas. The evolution of the pandemic also enables to collect post-confinement objective and subjective data, in order to analyse during the time the changes in the perception of the citizens. The second is because the methodology used to make the analysis includes both objective and perceptual metrics, and because of the inclusion of several key actors from other disciplines in the methodology.

Author Contributions: R.M.A.-P. conducted the recording campaign design, planning of the paper, writing and reviewing of the entire paper, and the conceptualization of the project. F.O. conducted the dissemination and education derivaties from the project Sons al Balcó and also wrote of Section 3.1. R.M. conducted the results analysis from the project and wrote Section 4. M.F. (Marc Freixes) prepared the setup for all the tests from project and wrote Section 3.2. X.B. conducted the design of the innovation strategy and citizen science communication strategy for the project and wrote Section 2. M.F. (Maria Foraster) set the noise and health framework and questionnaire design, wrote and reviewed parts of the paper and conceptualized the project. All authors have read and agreed to the published version of the manuscript.

Funding: The research that led to this contribution has been conducted thanks to funding from Secretaria d'Universitats i Recerca from the Departament d'Empresa i Coneixement (Generalitat de Catalunya) and Universitat Ramon Llull, under the grant 2020-URL-Proj-054 (Rosa Ma Alsina-Pagès).

Acknowledgments: The authors would like to thank all the participants of this first data collection campaign. The authors would also like to thank the Communications Departments of La Salle-URL and ISGlobal for their support in the dissemination. We would also like to thank Albert Cuesta for his article in Diari Ara, program "El Balcó" from Cadena SER Catalunya for the interview to RMAP, and finally, "Via Lliure" from RAC1 for the interview to RMAP and MF. All the media helped us to disseminate the project and collect more data for our project.

Conflicts of Interest: The authors declare no conflict of interest. 


\section{Abbreviations}

The following abbreviations are used in this manuscript:

$L_{\text {Aeq }} \quad$ Equivalent Level

UN United Nations

WHO World Health Organisation

\section{References}

1. WHO/Europe I Noise-Data and Statistics. Available online: www.euro.who.int/en/health-topics/ environment-and-health/noise/data-and-statistics (accessed on 6 September 2020).

2. Blanes, N.; Fons, J.; Houthuijs, D.; Swart, W.; de la Maza, M.; Ramos, M.; Castell, N.; van Kempen, E. Noise in Europe 2017: Updated Assessment; European Topic Centre on Air Pollution and Climate Change Mitigation (ETC/ACM): Bilthoven, The Netherlands, 2017.

3. Aletta, F.; Oberman, T.; Mitchell, A.; Tong, H.; Kang, J. Assessing the changing urban sound environment during the COVID-19 lockdown period using short-term acoustic measurements. Noise Mapp. 2020, 7, 123-134.

4. Basu, B.; Murphy, E.; Molter, A.; Basu, A.S.; Sannigrahi, S.; Belmonte, M.; Pilla, F. Effect of COVID-19 on noise pollution change in Dublin, Ireland. arXiv 2020, arXiv:2008.08993.

5. Asensio, C.; Aumond, P.; Can, A.; Gascó, L.; Lercher, P.; Wunderli, J.M.; Lavandier, C.; de Arcas, G.; Ribeiro, C.; Muñoz, P.; et al. A Taxonomy Proposal for the Assessment of the Changes in Soundscape Resulting from the COVID-19 Lockdown. Int. J. Environ. Res. Public Health 2020, 17, 4205.

6. Aletta, F.; Brinchi, S.; Carrese, S.; Gemma, A.; Guattari, C.; Mannini, L.; Patella, S.M. Analysing urban traffic volumes and mapping noise emissions in Rome (Italy) in the context of containment measures for the COVID-19 disease. Noise Mapp. 2020, 7, 114-122.

7. Asensio, C.; Pavón, I.; de Arcas, G. Changes in noise levels in the city of Madrid during COVID-19 lockdown in 2020. J. Acoust. Soc. Am. 2020, 148, 1748-1755.

8. Montano, W.; Gushiken, E. Lima soundscape before confinement and during curfew. Airplane flights suppressions because of Peruvian lockdown. JASA 2020, 148, 1824-1830.

9. COVID-19: The Quiet Project—Call for Measurements. Available online: www.ioa.org.uk/news/covid-19quiet-project-\%E2\%80\%93-call-measurements (accessed on 20 September 2019).

10. Grande Partecipazione all'iniziativa AIA di caratterizzazione dei Livelli Sonori Durante l'emergenza da Coronavirus. Available online: acustica-aia.it/grande-partecipazione-alliniziativa-aia-di-caratterizzazionedei-livelli-sonori-durante-lemergenza-da-coronavirus/ (accessed on 27 September 2019).

11. Locate Your Sound-Paesaggi Sonori Italiani Covid19. Available online: locateyoursound.com/en/ (accessed on 20 September 2019).

12. Scienzia sul Balcone. Available online: comunicazione.cnr.it/evento/254/scienzasulbalcone-misuriamo-ilrumore-intorno-a-noi (accessed on 19 October 2019).

13. The Coronavirus Quieted City Noise. Listen to What's Left. Available online: www.nytimes.com/interactive/ 2020/05/22/upshot/coronavirus-quiet-city-noise.html?smid=li-share (accessed on 6 September 2020).

14. Sounds from the Global Covid-19 Lockdown. Available online: citiesandmemory.com/covid19-sounds / (accessed on 20 September 2019).

15. Take Action for the Sustainable Development Goals. Available online: Www.un.org/ sustainabledevelopment/sustainable-development-goals / (accessed on 6 September 2020).

16. LimeSurvey Online Survey Tool. Available online: https://www.limesurvey.org/en/ (accessed on 12 October 2020).

17. Fine Uploader S3: Upload Directly to Amazon S3 from your Browser. Available online: https://blog. fineuploader.com / fine-uploader-s3-upload-directly-to-amazon-s3-from-your-browser-3d9dcdcc0f33 (accessed on 12 October 2020).

18. RecordRTC: WebRTC JavaScript Library for Audio+Video+Screen+Canvas (2D+3D Animation) Recording. Available online: https:/ / recordrtc.org/ (accessed on 12 October 2020). 
19. WebRTC: Real-Time Communication for the Web. Available online: https://webrtc.org/ (accessed on 12 October 2020).

20. Fine Uploader File Uploading. Available online: https: / / fineuploader.com/ (accessed on 12 October 2020). (C) 2020 by the authors. Licensee MDPI, Basel, Switzerland. This article is an open access article distributed under the terms and conditions of the Creative Commons Attribution (CC BY) license (http://creativecommons.org/licenses/by/4.0/). 